PROCEEDINGS OF THE

AMERICAN MATHEMATICAL SOCIETY

Volume 136, Number 8, August 2008, Pages 2895-2899

S 0002-9939(08)09247-2

Article electronically published on April 10, 2008

\title{
COMPACT WEIGHTED COMPOSITION OPERATORS ON THE HARDY SPACE
}

\author{
GAJATH GUNATILLAKE
}

(Communicated by Joseph A. Ball)

\begin{abstract}
A weighted composition operator $C_{\psi, \varphi}$ takes an analytic map $f$ on the open unit disk of the complex plane to the analytic map $\psi f \circ \varphi$, where $\varphi$ is an analytic map of the open unit disk into itself and $\psi$ is an analytic map on the open unit disk. This paper studies how the compactness of $C_{\psi, \varphi}$ depends on the interaction between the two maps $\psi$ and $\varphi$.
\end{abstract}

\section{INTRODUCTION}

Let $\varphi$ be an analytic map from the open unit disk $D$ into itself and let $\psi$ be an analytic map on the open unit disk. Then the weighted composition operator $C_{\psi, \varphi}$ on the Hardy space $H^{2}(D)$ is the operator that takes $f$ to $\psi f \circ \varphi$. That is, $\left(C_{\psi, \varphi} f\right)(z)=\psi(z) f(\varphi(z))$. If $\psi$ is identically 1 , this operator becomes a composition operator; this has been studied for about thirty-five years. However, less is known about more general weighted composition operators. If $\psi$ is a bounded analytic function on the open unit disk, then the multiplication operator $M_{\psi}$ where $M_{\psi}(f)(z)=\psi(z) f(z)$ becomes a bounded operator. The composition operator $C_{\varphi}$ where $C_{\varphi}(f)(z)=f(\varphi(z))$ is always bounded, and the weighted composition operator $C_{\psi, \varphi}$ is equal to $M_{\psi} C_{\varphi}$. Therefore, when the composition operator $C_{\varphi}$ is compact, the operator $C_{\psi, \varphi}$ will be compact. But if $\psi$ is unbounded, then the resulting multiplication operator is not bounded on $H^{2}(D)$ and hence the weighted composition operator $C_{\psi, \varphi}$ cannot be written as a product of a multiplication operator and a composition operator. Interestingly, it is possible for $C_{\psi, \varphi}$ to be compact with an unbounded $\psi$. Also when $M_{\psi}$ is bounded but neither of the operators $C_{\varphi}$ and $M_{\psi}$ are compact, it is still possible for $C_{\psi, \varphi}$ to be compact. For a bounded weight $\psi$ the compactness of the operator is discussed in Theorem 3.1 of [ [8], and this theorem depends on Alexandrov-Clark measures. Results in this paper depend on more direct methods. Also Lemma 3.3 of [1] discusses the compactness when the weight $\psi$ is smooth and $\varphi$ is a linear fractional map. This paper attempts to study the compactness of $C_{\psi, \varphi}$ without assuming that the weight function is bounded on the unit disk.

Received by the editors August 1, 2006, and, in revised form, June 2, 2007.

2000 Mathematics Subject Classification. Primary 47B32.

The research for this paper was undertaken in partial fulfillment of the requirements for the author's Ph.D. at Purdue University.

(C)2008 American Mathematical Society Reverts to public domain 28 years from publication 


\section{PRELIMINARIES}

The Hardy space $H^{2}(D)$ is the set of functions analytic on the open unit disk for which

$$
\sup _{0<r<1} \int_{0}^{2 \pi}\left|f\left(r e^{i \theta}\right)\right|^{2} \frac{d \theta}{2 \pi}<\infty
$$

If $f$ is in the space $H^{2}(D)$, then it can be extended to the unit circle almost everywhere. More precisely, $f^{*}\left(e^{i \theta}\right)=\lim _{r \rightarrow 1} f\left(r e^{i \theta}\right)$ exists for almost all $\theta$, and the mapping $f \rightarrow f^{*}$ is an isometry of $H^{2}(D)$ into a closed subspace of $L^{2}(d \theta / 2 \pi)$. For a proof and more detailed discussions see [3, page 10] and [4, page 21]. But in Hardy space literature $f^{*}$ is seldom used. With a slight abuse of notation $f$ is used instead of $f^{*}$ on the unit circle. Moreover the mapping $f \rightarrow f^{*}$ behaves nicely with respect to composition. That is, $(f \circ \varphi)^{*}=f^{*} \circ \varphi^{*}$ almost everywhere [3, page 31]. If $f$ is the constant function 1 , then $C_{\psi, \varphi}(f)=\psi$, and in order for the operator $C_{\psi, \varphi}$ to be bounded on $H^{2}(D)$ it is clear that $\psi$ must be in the space $H^{2}(D)$. Therefore we will always make the assumption that the weight function $\psi$ is in the Hardy space $H^{2}(D)$.

The following lemma was inspired by a similar result for unweighted composition operators [3, Proposition 3.11].

Lemma 1. Let $C_{\psi, \varphi}$ be a bounded operator on $H^{2}(D)$. Then $C_{\psi, \varphi}$ is compact if and only if whenever $\left\{f_{n}\right\}$ is a bounded sequence in $H^{2}(D)$ and $f_{n} \rightarrow 0$ uniformly on compact subsets of $D$ then $C_{\psi, \varphi}\left(f_{n}\right) \rightarrow 0$ in $H^{2}(D)$.

A proof of this lemma can be found in [6, page 17]. We now use the previous lemma to prove the following theorem.

Theorem 1. Let $C_{\psi, \varphi}$ be a bounded operator on $H^{2}(D)$. Suppose $\varphi$ is continuous up to the boundary of the unit disk. Let $Z=\{\zeta:|\varphi(\zeta)|=1, \zeta$ on the unit circle $\}$. Assume that $\psi(\zeta)=0$ for each $\zeta$ in $Z$ and also that $\psi$ is continuous at $\zeta$. Then the operator $C_{\psi, \varphi}$ is compact.

Proof. Let $\left\{f_{n}\right\}$ be a bounded sequence in $H^{2}(D)$ which approaches 0 uniformly on compact subsets of the open unit disk. We will show that $C_{\psi, \varphi}\left(f_{n}\right)$ approaches 0 in $H^{2}(D)$. This will prove that the operator is compact. The unweighted composition operator $C_{\varphi}$ is also bounded; therefore there is a positive $M$ such that $\left\|f_{n}\right\|^{2}\left\|C_{\varphi}\right\|^{2}<M$.

Suppose $\zeta$ is in $Z$. Then $\psi(\zeta)=0$ and $\psi$ is continuous at $\zeta$. Therefore there is $t_{\zeta}>0$ such that $\left|\psi\left(\zeta e^{i \theta}\right)\right|^{2}<\epsilon / 2 M$ whenever $-t_{\zeta}<\theta<t_{\zeta}$. Let $U_{\zeta}$ denote the set $\left\{\zeta e^{i \theta}:-t_{\zeta}<\theta<t_{\zeta}\right\}$ and $U=\bigcup_{\zeta \in Z} U_{\zeta}$. Suppose $T$ denotes the unit circle and $V$ denotes $T-U$ :

$$
\begin{aligned}
\left\|C_{\psi, \varphi}\left(f_{n}\right)\right\|^{2} & =\int_{T}\left|\psi\left(e^{i \theta}\right) f_{n}\left(\varphi\left(e^{i \theta}\right)\right)\right|^{2} \frac{d \theta}{2 \pi} \\
& =\int_{U}\left|\psi\left(e^{i \theta}\right) f_{n}\left(\varphi\left(e^{i \theta}\right)\right)\right|^{2} \frac{d \theta}{2 \pi}+\int_{V}\left|\psi\left(e^{i \theta}\right) f_{n}\left(\varphi\left(e^{i \theta}\right)\right)\right|^{2} \frac{d \theta}{2 \pi} .
\end{aligned}
$$


Since $\left|\psi\left(e^{i \theta}\right)\right|^{2}<\epsilon / 2 M$ for $e^{i \theta}$ in $U$ we get

$$
\begin{aligned}
\int_{U}\left|\psi\left(e^{i \theta}\right)\right|^{2}\left|f_{n}\left(\varphi\left(e^{i \theta}\right)\right)\right|^{2} \frac{d \theta}{2 \pi} & \leqslant(\epsilon / 2 M) \int_{U}\left|f_{n}\left(\varphi\left(e^{i \theta}\right)\right)\right|^{2} \frac{d \theta}{2 \pi} \\
& \leqslant(\epsilon / 2 M)\left\|C_{\varphi}\left(f_{n}\right)\right\|^{2} \\
& \leqslant(\epsilon / 2 M)\left\|f_{n}\right\|^{2}\left\|C_{\varphi}\right\|^{2} \\
& \leqslant \epsilon / 2 .
\end{aligned}
$$

Since $V$ is a compact subset of the unit circle and $\varphi$ is continuous on the unit circle, $\varphi(V)$ is a compact subset of the open unit disk. Therefore the sequence $\left\{f_{n}\right\}$ converges to zero uniformly on $\varphi(V)$. Thus for all $e^{i \theta}$ in $V$ and all $n$ large enough,

$$
\left|f_{n}\left(\varphi\left(e^{i \theta}\right)\right)\right|^{2} \leqslant \frac{\epsilon}{2\|\psi\|^{2}} .
$$

Therefore,

$$
\begin{aligned}
\int_{V}\left|\psi\left(e^{i \theta}\right)\right|^{2}\left|f_{n}\left(\varphi\left(e^{i \theta}\right)\right)\right|^{2} \frac{d \theta}{2 \pi} & \leqslant \frac{\epsilon}{2 \| \psi||^{2}} \int_{V}\left|\psi\left(e^{i \theta}\right)\right|^{2} \frac{d \theta}{2 \pi} \\
& \leqslant \frac{\epsilon}{2} ;
\end{aligned}
$$

hence by (2),

$$
\int_{T}\left|\psi\left(e^{i \theta}\right)\right|^{2}\left|f_{n}\left(\varphi\left(e^{i \theta}\right)\right)\right|^{2} \frac{d \theta}{2 \pi}<\epsilon
$$

for all $n$ large enough. Thus $\lim _{n \rightarrow \infty} C_{\psi, \varphi}\left(f_{n}\right)=0$ in norm. Now the compactness of $C_{\psi, \varphi}$ follows from Lemma 1 .

Theorem 1 can be used to construct some interesting examples. We first state the following lemma. The proof can be found in [10, page 31].

Lemma 2. If $\varphi$ is a univalent map of the unit disk into itself and if $\varphi(D)$ contains a disc in $D$ that is tangent to the unit circle, then the composition operator $C_{\varphi}$ cannot be compact on $H^{2}(D)$.

Example 1. Let $\varphi(z)=(z+1) / 2$. The image of the unit circle under the map $\varphi$ is tangent to the unit circle at the point $(1,0)$. Therefore by Lemma 2 the unweighted composition operator $C_{\varphi}$ is not compact. Now let $\psi(z)=1-z$. Then the multiplication operator $M_{\psi}$ is bounded and therefore $C_{\psi, \varphi}=M_{\psi} C_{\varphi}$ is bounded. By Theorem 1 the operator $C_{\psi, \varphi}$ is compact.

Lemma 3. If $\varphi(z)=(z+1) / 2$ and $\psi(z)=((1-z) /(1+z))^{1 / 4}$, then the operator $C_{\psi, \varphi}$ is bounded.

Proof. Let $f$ be in $H^{2}$. The image of the unit circle under the map $\varphi$ is a circle of radius $1 / 2$ tangent to the unit circle at 1 . Also $\varphi$ is one to one. Therefore it is possible to choose an open arc $V$ of the unit circle containing the point 1 such that $\left|\varphi\left(e^{i \theta}\right)\right|<3 / 4$ for every $e^{i \theta}$ outside $V$. Let $U$ consist of all $e^{i \theta}$ that do not belong to $V$. Now,

$$
\begin{aligned}
\left\|C_{\psi, \varphi}(f)\right\|^{2} & =\int_{T}\left|\psi\left(e^{i \theta}\right)\right|^{2}\left|f\left(\varphi\left(e^{i \theta}\right)\right)\right|^{2} \frac{d \theta}{2 \pi} \\
& =\int_{U}\left|\psi\left(e^{i \theta}\right)\right|^{2}\left|f\left(\varphi\left(e^{i \theta}\right)\right)\right|^{2} \frac{d \theta}{2 \pi}+\int_{V}\left|\psi\left(e^{i \theta}\right)\right|^{2}\left|f\left(\varphi\left(e^{i \theta}\right)\right)\right|^{2} \frac{d \theta}{2 \pi} .
\end{aligned}
$$


If $e^{i \theta}$ is in $U$, then $\left|\varphi\left(e^{i \theta}\right)\right|<3 / 4$. Hence $\left|f\left(\varphi\left(e^{i \theta}\right)\right)\right| \leq \| f|| / \sqrt{1-9 / 16}$, and if $e^{i \theta}$ is in $V$, then $\left|\psi\left(e^{i \theta}\right)\right|<K$ for some $K$. Now by (5),

$$
\left\|C_{\psi, \varphi}(f)\right\|^{2} \leq \frac{16}{7}\|f\|^{2} \int_{U}\left|\psi\left(e^{i \theta}\right)\right|^{2} \frac{d \theta}{2 \pi}+K^{2} \int_{V}\left|f\left(\varphi\left(e^{i \theta}\right)\right)\right|^{2} \frac{d \theta}{2 \pi} .
$$

Since $\psi$ is in $H^{2}$ and the integral of $|f \circ \varphi|^{2}$ over $V$ is smaller than $\|f\|^{2}\left\|C_{\varphi}\right\|^{2}$,

$$
\left\|C_{\psi, \varphi}(f)\right\|^{2} \leq\|f\|^{2}\left(\frac{16}{7}\|\psi\|^{2}+K^{2}\left\|C_{\varphi}\right\|^{2}\right) .
$$

Thus $C_{\psi, \varphi}$ is bounded.

Example 2. Let $\varphi(z)=(z+1) / 2$ and $\psi(z)=((1-z) /(1+z))^{1 / 4}$. Then by Lemma 3, $C_{\psi, \varphi}$ is a bounded operator. The only place where $|\varphi(\zeta)|=1$ is when $\zeta=1$ and $\psi$ is continuous on the unit circle. Now Theorem 1 tells us that the operator $C_{\psi, \varphi}$ is compact.

It is interesting to note that in the above example the composition operator $C_{\varphi}$ is not compact and the multiplication operator $M_{\psi}$ is not even bounded, but the weighted composition operator $C_{\psi, \varphi}$ is compact.

In Example1, we began with a non-compact composition operator $C_{\varphi}$ and ended up with a compact weighted composition operator $C_{\psi, \varphi}$ by choosing an appropriate weight. A natural follow up question would be, can we do the opposite? That is, given a compact composition operator $C_{\varphi}$, can we choose a weight $\psi$ so that the operator $C_{\psi, \varphi}$ is non-compact (but bounded)? The answer, at least in some cases, is, interestingly, no.

Theorem 2. Suppose $\overline{\varphi(D)}$ is contained in the open unit disk D. Then for any $\psi$ in the Hardy space $H^{2}(D)$ the weighted composition operator $C_{\psi, \varphi}$ is compact.

Proof. We will show that $\left.|| C_{\psi, \varphi}\right|_{z^{n} H^{2}(D)} \|$ tends to zero as $n$ tends to infinity. This will prove the compactness of the operator. Since $\overline{\varphi(D)}$ is contained in the open unit disk, $|\varphi(\zeta)| \leq r$ for some $r<1$ for almost all $\zeta$ on the unit circle. Suppose $f$ is in the subspace $z^{n} H^{2}(D)$; then $f(z)=z^{n} g(z)$ for some $g$ in $H^{2}(D)$ with $\|f\|=\|g\|$.

$$
\begin{aligned}
\left\|C_{\psi, \varphi} f\right\|^{2} & =\int_{T}\left|\psi\left(e^{i \theta}\right)\right|^{2}\left|\varphi\left(e^{i \theta}\right)\right|^{2 n}\left|g\left(\varphi\left(e^{i \theta}\right)\right)\right|^{2} \frac{d \theta}{2 \pi} \\
& \leq r^{2 n} \int_{T}\left|\psi\left(e^{i \theta}\right)\right|^{2}\left|g\left(\varphi\left(e^{i \theta}\right)\right)\right|^{2} \frac{d \theta}{2 \pi} .
\end{aligned}
$$

But

so that

$$
\left|g\left(\varphi\left(e^{i \theta}\right)\right)\right| \leq\|g\| \frac{1}{\sqrt{1-r^{2}}}
$$

$$
\left\|C_{\psi, \varphi} f\right\|^{2} \leq r^{2 n} \frac{\|g\|^{2}}{1-r^{2}} \int_{T}\left|\psi\left(e^{i \theta}\right)\right|^{2} \frac{d \theta}{2 \pi}
$$

Now, $\psi$ is in the Hardy space and $\|g\|=\|f\|$; thus,

$$
\left\|C_{\psi, \varphi} f\right\|^{2} \leq \frac{r^{2 n}}{1-r^{2}}\|\psi\|^{2}\|f\|^{2} .
$$

Now we have

$$
\left\|\left.C_{\psi, \varphi}\right|_{z^{n} H^{2}(D)}\right\|^{2} \leq \frac{r^{2 n}}{1-r^{2}}\|\psi\|^{2}
$$


Therefore $\left.|| C_{\psi, \varphi}\right|_{z^{n} H^{2}(D)}||$ tends to zero as $n$ tends to infinity; thus $C_{\psi, \varphi}$ is compact.

Can compactness of $C_{\psi, \varphi}$ force the compactness of $C_{\varphi}$ ? The answer is yes if $|\psi|$ is essentially bounded below on the unit circle, for then the operator $M_{\psi}$ is bounded below and compactness of $M_{\psi} C_{\varphi}$ implies compactness of $C_{\varphi}$.

The following example shows that the continuity hypothesis on the weight $\psi$ in Theorem 1 cannot be completely removed.

Example 3. Let $\psi(z)=e^{(z+1) /(z-1)}$ and let $\varphi(z)=(z+1) / 2$. The radial limit $\lim _{r \rightarrow 1^{-}} \psi(r)=0$; therefore $\psi(1)=0$ and the only place $|\varphi(z)|=1$ is $z=1$. Since $\psi$ has modulus 1 almost everywhere on the unit circle, $M_{\psi}$ is an isometry. Thus $M_{\psi} C_{\varphi}$ cannot be compact.

\section{ACKNOWLEDGMENTS}

The research for this paper was undertaken in partial fulfillment of the requirements for the author's Ph.D. at Purdue University. The author thanks his thesis advisor, Professor Carl Cowen, for his guidance throughout this research.

\section{REFERENCES}

[1] P. Bourdan, D. Levi, S. K. Narayan and J. H. Shapiro, Which linear-fractional composition operators are essentially normal? JMAA 280 (2003), 30-53. MR.1972190(2003m:47042)

[2] J. Clifford and M. Dabkowski, Singular values and Schmidt pairs of composition operators on the Hardy Space, JMAA 305 (2005), 183-196. MR.2128121 (2005m:47045)

[3] C. Cowen and B. MacCluer, Composition Operators on Spaces of Analytic Functions, CRC Press, Boca Raton, FL, 1995. MR1397026 (97i:47056)

[4] P. Duren, Theory of $H^{p}$ Spaces, Academic Press, New York-London, 1970. MR0268655 $(42: 3552)$

[5] F. Forelli, The isometries of $H^{p}$, Canad. J. Math. 16 (1964), 721-728. MR0169081 (29:6336)

[6] G. Gunatillake, Weighted Composition Operators, Doctoral Dissertation, Purdue University, 2005.

[7] P. Halmos, A Hilbert Space Problem Book, Springer-Verlag, New York, 1967. MR675952 (84e:47001)

[8] T. Kriete and J. Moorhouse, Linear relations in the Calkin algebra for composition operators, Trans. Amer. Math. Soc. 359 (2007), 2915-2944. MR2286063

[9] W. Rudin, Real and Complex Analysis, McGraw-Hill, New York, 1966. MR0210528(35:1420)

[10] J. Shapiro, Composition Operators and Classical Function Theory, Springer-Verlag, New York, 1993. MR1237406 (94k:47049)

[11] J. Shapiro and W. Smith, Hardy spaces that support no compact composition operators, J. Functional Anal. 205 (2003), 62-89. MR2020208 (2004h:30041)

Department of Mathematics, American University of Sharjah, Sharjah, United Arab EMIRATES

E-mail address: mgunatillake@aus.edu 